This item was submitted to Loughborough's Research Repository by the author.

Items in Figshare are protected by copyright, with all rights reserved, unless otherwise indicated.

\title{
Optimizing communication and computation for multi-UAV information gathering applications
}

\section{PLEASE CITE THE PUBLISHED VERSION}

https://doi.org/10.1109/TAES.2017.2761139

\section{PUBLISHER}

(C) Institute of Electrical and Electronics Engineers (IEEE)

VERSION

AM (Accepted Manuscript)

LICENCE

CC BY-NC-ND 4.0

\section{REPOSITORY RECORD}

Thammawichai, Mason, Sujit P. Baliyarasimhuni, Eric C. Kerrigan, and Joao Borges de Sousa. 2018. "Optimizing Communication and Computation for Multi-uav Information Gathering Applications". figshare. https://hdl.handle.net/2134/27955. 


\title{
Optimizing Communication and Computation for Multi-UAV Information Gathering Applications
}

\author{
Mason Thammawichai, Student Member, IEEE, Sujit P. Baliyarasimhuni, Member, IEEE, \\ Eric C. Kerrigan, Member, IEEE, and João B. Sousa, Member, IEEE
}

\begin{abstract}
Typical mobile agent networks, such as multi-UAV systems, are constrained by limited resources: energy, computing power, memory and communication bandwidth. In particular, limited energy affects system performance directly, such as system lifetime. Moreover, it has been demonstrated experimentally in the wireless sensor network literature that the total energy consumption is often dominated by the communication cost, i.e. the computational and the sensing energy are small compared to the communication energy consumption. For this reason, the lifetime of the network can be extended significantly by minimizing the communication distance as well as the amount of communication data, at the expense of increasing computational cost. In this work, we aim at attaining an optimal trade-off between the communication and the computational energy. Specifically, we propose a mixed-integer optimization formulation for a multihop hierarchical clustering-based self-organizing UAV network incorporating data aggregation, to obtain an energy-efficient information routing scheme. The proposed framework is tested on two applications, namely target tracking and area mapping. Based on simulation results, our method can significantly save energy compared to a baseline strategy, where there is no data aggregation and clustering scheme.
\end{abstract}

Index Terms-Unmanned aerial vehicles, Multi-agent systems, Cooperative systems, Optimal control, Optimization

\section{INTRODUCTION}

Inexpensive mobile agents, such as unmanned aerial vehicles (UAVs), are useful for several remote monitoring applications such as agriculture [1], geology [2], ecology [3] and forestry [4]. The viability of UAVs for scientific and nonmilitary applications are due to reduced cost of the UAVs, low sensor cost and ease in handling. Typically, these applications are of large scale and the mission time can be shortened by introducing multiple UAVs.

Central to these applications is the necessity to have a human-in-the-loop (HITL) capability that increases situational awareness and operator autonomy to modify missions dynamically. For HITL, UAVs have to gather and disseminate information periodically to the operator who may be located at a distant (base station) from the operational arena. Typical information required at the base station is aerial footage [5],

Mason Thammawichai is with the Department of Aeronautics, Imperial College London, London SW7 2AZ, UK.

Sujit P. Baliyarasimhuni is with IIIT-Delhi, New Delhi - 110020, India, and Department of Aeronautical and Automotive Engineering, Loughborough University, Leicestershire, UK LE11 3TU.

Eric C. Kerrigan is with the Department of Electrical \& Electronic Engineering and the Department of Aeronautics, Imperial College London, London SW7 2AZ, UK.

João B. Sousa is with the Department of Electrical and Computer Engineering, University of Porto, Portugal - 4200-465. which is a communication intensive operation consuming considerable energy. Unfortunately, low cost UAVs have limited flight time due to battery/fuel capacity. Hence, there is a need to find different mechanisms by which flight time endurance can be increased. One way is to use gliders that take advantage of the updrafts to soar for long endurance [6]. However, during soaring it is highly difficult to maintain a good resolution of the terrain due to varying UAV height for mapping or surveillance applications. Instead, we propose to optimize the energy consumed by various units in a given aircraft to increase the flight time and hence the UAV team mission time.

For many applications [1], [4], it is necessary that a UAV must fly at a constant speed and maintain a prescribed height. Under these conditions, the major energy consumption units are propulsion, sensing, computation and communication. On average, the power consumed during flight is approximately constant. One of the low cost UAV used for civilian applications is a Skywalker 1880mm wingspan [7]. A typical motor used for this vehicle is T-motor 2820 [8] that consumes $668 \mathrm{~W}$ at full thrust. During the mission, typical thrust is between $50-60 \%$ and hence the consumption is $\approx 335-401 \mathrm{~W}$. The sensing and the computational units also consume constant power and they are not a function of the vehicle velocity or altitude. Typical, popular on board embedded computers used in low cost UAVs are Raspberry Pi3 [9], ODROID xu4 [10], and NVIDIA Jetson K1 [11]. These computers have a power consumption of approximately $8-12 \mathrm{~W}(\mathrm{~J} / \mathrm{s}), 10-12 \mathrm{~W}(\mathrm{~J} / \mathrm{s})$ and $6-10 \mathrm{~W}(\mathrm{~J} / \mathrm{s})$, respectively which is approximately constant. However, the energy expended by the communication depends on (i) the amount of data to be transmitted, (ii) the distance between a vehicle" and the base station and (iii) the number of vehicles transmitting data to the base station. Moreover, the communication cost is far greater than the sensing and computational energy. For example, a typical sensor node consumes $1 \mathrm{~nJ}-1 \mu \mathrm{J} / \mathrm{sample}$, roughly $1 \mathrm{pJ} /$ instruction for computation, while communicating via radio frequency (RF) at the cost of $100 \mathrm{~nJ}-50 \mu \mathrm{J}$ per bit [12]. Consider another example, where we assume that only one UAV is broadcasting an image of $1280 \times 720$ pixels to the base station at a distance of $1000 \mathrm{~m}$, then the communication cost for transmission is 2306 Joules which is far higher than the sensing and computing costs. Clearly, we can see that the communication cost dominates over propulsion and computation. Moreover, the costs associated with propulsion and computation are approximately constant on average, while the communication cost is a functions of distance and the amount of information to be broadcast. Hence, it is better for the UAVs to cooperate with 
each other to minimize the team communication energy by performing computation on-board such that the amount of data to be transmitted is minimized. That is, optimally selecting (a) which vehicles should be the computing nodes and (b) determining how many vehicles are required to communicate with the base station. In this paper, we propose a general Mixed Integer Nonlinear Program (MINLP) that determines an optimal solution to (a) and (b).

\section{A. Related Work}

Similar to our Multi-UAV information gathering problem, the goal of a Wireless Sensor Network (WSN) is to maximize network lifetime while delivering raw data to the sink (base station) [13]. In order to maximize the lifetime of a network, data aggregation techniques have been proposed for WSNs [14], [15]. Data correlations between different sensor nodes can be exploited to minimize the number of sensors sending the data to the base station [16]. A compressed sensing technique to reduce the data volume to be transmitted was proposed in [17].

Hierarchical Network Routing is also one of the techniques in prolonging a network lifetime. For this approach, the nodes are grouped into clusters and the cluster-head for each group is selected based on various election algorithms [18]. The cluster head is responsible for aggregation, compression and forwarding data to the base station. In [19], a stochastic scheme is used to determine whether a node will become a clusterhead in each decision making round. The stochastic scheme was improved in [20] by using global information of the network to determine an optimal number of cluster heads via a centralized control at the base station. A chain-based protocol was proposed in [21], where the nodes are only allowed to communicate with nearby nodes and take turns to transmit data to the base station. A hierarchical data aggregation technique where sensor nodes were grouped into clusters was proposed in [22]. A local aggregator (LA) for each cluster was selected, then a set of master aggregators (MAs) were selected based on LAs. To select MAs, an integer program is solved such that the total communication energy is minimized, while performing minimum aggregation computation, such as finding an average or a maximum. For this work, we adopt a hierarchical clusterbased data aggregation technique from the WSN literature, but the topology of the network and the number of MAs are dynamically decided.

Another approach is to have a mobile sensing node collect data from the nodes to reduce the communication overload [23]-[26]. Since the UAVs are mobile, using another UAV to collect data from the surveying UAVs is not an ideal approach. However, similar to WSN data aggregation, the UAVs can perform computations on board to produce concise data and periodically transmit to the base station, as in [27] for an image processing application. Data transmission to the base station can be performed either directly or through a UAV relay network [28], [29]. Therefore, in this work, we propose a self-organizing network topology that allows data aggregation as well as a multi-hop information routing pattern.

Nonetheless, the more related work to ours is the work of [30]. In particular, they proposed the solution to the communication-aware information gathering problem. That is to dynamically decide the information flow between a pair of robots with the objective of maximizing an information value of the communication subjected to a bandwidth constraint. However, our work is different, as our network model allows multiple data type flows, while theirs assume one data type, which then increases the complexity of the problem significantly. Specifically, our problem cannot simply be modelled as a network flow problem, which can then be solved using a linear program. Therefore, we propose a general MINLP optimization framework to solve the problem of multi-UAV information gathering applications with a multiple flows of more than one data type.

A UAV with sensing capabilities can be applied to perform target tracking due to its adaptability, scalability and better performance than a static wireless sensor network. However, most of the work on UAV target tracking applications only focus on the target tracking accuracy, while the communication and computation energy consumption has been neglected [31][34]. Hence, this work aims to incorporate both the communication and computation energy consumption into a multiUAV target tracking application. Target tracking algorithms are based on target state estimation. By combining multiple sensor readings, which originated from different moments in time and distances from the UAVs, a more accurate state estimate can be obtained [35]. Precisely, the tracking objective is to maximize the information contribution [35], [36] from each node. In general, it has been shown that the measurement obtained from the most distant node does not contribute much to the target tracking accuracy. Therefore, it would be energy-efficient to select only the subset of the UAVs to be tracking nodes. The problem of deciding a subset of tracking sensor nodes could be formulated as an MINLP as in [37], where the observation covariance depends on the distance, i.e. the further away from the target, the less accurate the measurement. Therefore, in this work, we include the information contribution constraint to our optimal control formulation for a target tracking application.

Energy-efficiency in target tracking applications has been extensively studied in the WSN area; see [38] for an up-todate survey on the topic. In the WSN literature, two classes of methods have been used to achieve energy-efficiency in collaborative WSN-based target tracking applications: a sensingrelated method and a communication-related method. The sensing-related method uses information-driven [39], [40] and data filtering techniques [41] to determine which sensor should sense the information and to which sensor the data should be sent in order to maximize the information value, i.e. target tracking accuracy. The communication-related approach aims at optimizing routing and aggregation techniques [42] as well as network self-organization [43]-45] to extend the network lifetime. Note that, it has to be mentioned that an optimal energy management scheme cannot be obtained by applying only one particular approach, since they are highly dependent on each other. Therefore, for this work, we are interested in determining the trade-offs between these techniques such that an optimal energy efficient management scheme is achieved.

UAVs have been used for mapping applications [1], [46][48]. However, the focus of mapping applications using UAVs 
has been on improving the accuracy of the acquired images. In some applications, the objective is to determine minimum energy cost path for UAVs. In [49], the objective for the UAV is to visit a set of pre-defined target locations. The determined path must minimize the total energy consumed in visiting the targets. In [50], the objective is to develop multi-UAV exploration strategies under limited battery constraints. In [51], a multi-UAV cooperative system using behaviors was developed to efficiently explore a region with the constraint that the UAVs have limited energy. In most of the above UAV mapping applications, the issue of optimizing communication energy to enhance mission time is not considered. In our formulation, we want to optimize the energy consumed by communication and computation components, so that the mission duration can be increased. This aspect has not be adequately addressed in the UAV mapping literature.

\section{B. Contribution}

This paper proposes a simple optimal control problem for mobile agent systems with the objective of minimizing the communication and the computation energy. Particularly, we present an MINLP formulation for a multi-hop hierarchical cluster-based self-organizing UAV network to attain an energyefficient reporting mechanism. The main contributions of this work are:

- A general MINLP optimization framework for a multiUAV network to optimally trade-off between the communication and the computational energy is presented. That is, to dynamically determine: (i) the optimal number of agents to communicate to the base station, (ii) the role of each UAV: a sensor, a relay or an aggregator, (iii) the communication links among the UAVs to obtain an energy-efficient information routing network with data aggregation.

- Our data aggregation network model exploits three beneficial characteristics: (i) a self-organizing network, (ii) a multi-hop network, and (iii) a hierarchical clustering network.

- A generalised data aggregation network model that allows multiple flows of more than one data type within the network.

- Two information gathering applications, namely target tracking and area mapping are addressed by our proposed optimal control framework to illustrate both the correctness and the effectiveness in trading off communication and computation energy.

- Simulation results show an energy saving of up to $40 \%$ for target tracking and $60 \%$ for area mapping when comparing the performance of our MINLP formulation with a baseline approach, where there is no data aggregation and clustering scheme.

\section{Outline of Paper}

The rest of the paper is organized as follows: In Section II the application details are presented. Details on problem assumptions, system models and variable definitions are given in Section III. The optimal control problem formulation is presented in Section IV The optimal control problem is applied to target tracking and mapping applications in Section $[\mathrm{V}$ as well as simulation results. We conclude in Section VI

\section{Application Details}

For this paper, we are looking at the scenerio where a team of $n$ UAVs is given a mission to either pursue a single target or survey an area of interest (AOI) and needs to periodically send the data back to the base station.

\section{A. System Assumptions}

We will assume that at each decision making time interval, each UAV (node) $i \in N:=\{1, \ldots, n\}$ has the same capability of sensing, data aggregation and communication functions, where $n$ is the total number of UAVs in the fleet. A UAV can reach any UAV using one-hop communication. A sensing UAV periodically senses a target/AOI, i.e. information (a data packet) is generated at a constant rate, and hence, the energy consumed by the sensor is constant. We assume that the UAVs are flying at constant altitude having constant speed and there are no wind disturbances. The power consumed by the propulsion unit during level flight is given by the relation

$$
P_{\text {prop }}=\frac{C_{D}}{C_{L}^{3 / 2}} \sqrt{\frac{2 R g^{3}}{\rho}} \frac{m^{\frac{3}{2}}}{b},
$$

where $C_{D}$ is the drag coefficient, $C_{L}$ is the lift coefficient, $R$ is the aspect ratio of the aircraft, $g$ is the gravity constant, $\rho$ is the air density, $m$ is the mass of the aircraft and $b$ is the wing span [52]. As we can see from the relation that for a level flight, all the quantities associated with $P_{\text {prop }}$ are constant. Further, since we assume that the UAV is flying at a fixed altitude, the lift and drag cooefficients that depend on the velocity of the aircraft are also constant. Hence we assume that the energy consumed by the propulsion unit is constant under these assumptions. As the sensor and propulsion energy consumption is constant, including them in the formulation does not affect the decisionmaking. Hence, we do not consider them in our formulation. The information can be of different types, therefore our model can be thought of as either a single source or multiple sources with different data types. For simplicity, we will consider a system with only one base station to report the data. Note that extension to multiple sink nodes (base stations) is relatively straightforward.

\section{B. UAV as a Mobile Computing Node}

For this work, a UAV will be modeled as a mobile computing node, which is composed of three primary modules: a sensor module, a processing module and a wireless communication module, where interactions between modules are shown in Figure 11. The main activities of the sensor module includes sensing, analog to digital conversion (ADC) and signal modulation. The processing module is responsible for data processing, sensor control as well as the communication protocol. The wireless communication module is used for transmitting and receiving. We will assume that there exists a 


\begin{tabular}{|c|c|c|c|c|c|}
\hline Symbol & Description & Symbol & Description & Symbol & Description \\
\hline$N$ & Set of all UAVs (nodes) & $n$ & Total number of nodes & $C$ & Communication link matrix \\
\hline$c$ & Communication link assignment & $M$ & Set of all data types & $m$ & Total number of data types \\
\hline$\lambda$ & Average data transmitting rate & $\bar{\lambda}$ & Sensing rate & $\epsilon$ & Sufficiently small constant \\
\hline$|G|$ & Number of sensors of a data type & $a$ & Aggregator assignment & $\gamma$ & Aggregator ratio \\
\hline$B$ & Communication bandwidth & $L$ & Packet length & $h$ & Decision time interval \\
\hline$E$ & Energy consumption & $d$ & Distance between nodes & $e$ & Energy state vector \\
\hline$\phi$ & Inertial position vector & $x$ & Position in $\mathrm{x}$-axis & $y$ & Position in $\mathrm{y}$-axis \\
\hline$v / V$ & Speed/speed vector & $\phi$ & Heading angle & $r$ & Distance/range \\
\hline$X$ & State of the system & $u$ & Control input & $\pi$ & Information contribution \\
\hline$H$ & Observation matrix & $R$ & Measurement covariance matrix & $F_{0}$ & State transition matrix \\
\hline$w_{0}$ & Process noise vector & $Q_{0}$ & Noise covariance matrix & $Z$ & Measurement vector \\
\hline$\nu$ & Measurement noise vector & $K$ & Distance-independent coefficient & $Q$ & Information matrix \\
\hline$P$ & Covariance error matrix & $\hat{q}$ & Information state vector & $S$ & Set of sensor nodes \\
\hline$W$ & Width of a region & $T$ & Length of a region & $\zeta$ & Overlap factor \\
\hline$N_{\ell}$ & Total number of lanes & $\ell$ & Lane & $\omega$ & Waypoint \\
\hline$\tau$ & Transition boundary & $\chi$ & Entry angle & $\Phi$ & Heading angle vector \\
\hline \multicolumn{6}{|c|}{ Subscritpts/Superscripts } \\
\hline$i, j$ & UAV (node) & 0 & Source (target) node/initial state & $n+1$ & Sink node (base station) \\
\hline$z$ & Data type & $c$ & Communication & $s$ & Sensing \\
\hline$p$ & Processing & $t$ & Transmitting & $r$ & Receiving \\
\hline$\beta$ & Path loss exponent & + & Next state & $k$ & Decision making round \\
\hline$\kappa$ & Lane index & $b$ & Bottom & $d$ & Desired heading angle \\
\hline
\end{tabular}

TABLE I: Notations

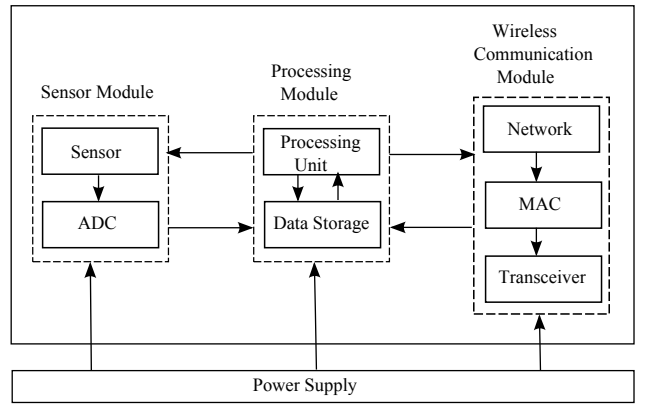

Fig. 1: The architecture of a mobile computing node (adapted from [18])

medium access control (MAC) protocol, which allows a UAV to communicate with other UAVs and the base station within a transmission range.

\section{UAV Role Assignment}

Following the works of [14] and [53], we will assume that the UAVs can be assigned to one or more of the following roles at each time interval: (i) a sensor, which observes the target/AOI (called node 0), via a sensor and produces the data which will be relayed to the base station (called node $n+$ 1), (ii) a relay, which simply relays its own data to the next level node without any processing, or (iii) an aggregator, which receives one or more data from other nodes, then aggregates the data of the same type to produce a single data point and sends the aggregated data to the next level node.

\section{Aggregation Network Topology}

Figure 2 illustrates the information flow in an aggregation network topology. In particular, the data obtained from the source (target/AOI) can be processed within the aggregator

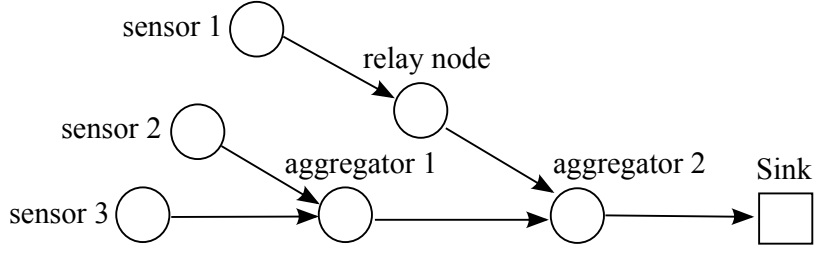

Fig. 2: Information Flow in an Aggregation Network Topology

or passed along the relay node and routed to the sink (base station). Note that, in this work, the network topology is dynamic, which differs from others in the WSN literature, i.e. the roles of the UAVs are decided at each time interval. Moreover, only data of the same type is allowed to be compressed/aggregated.

\section{DynamiC Model With COnstraints}

\section{A. Communication Model and Constraints}

Let $C:=\left[c_{i j z}\right]$ denote a communication link matrix, i.e. $c_{i j z}=1$ if node $i$ transmits data of type $z$ to node $j$ for $i, j \in N^{+}:=N \cup\{0, n+1\}, z \in M:=\{1, \ldots, m\}$. Note that $c_{0 i z}=1$ if node $i$ is a sensor of data type $z$ and $c_{i(n+1) z}=1$ if node $i$ sends data type $z$ to the base station. The communication link matrix $C$ is subject to

$$
\begin{array}{ll}
c_{i j z} \in\{0,1\}, & \forall i \in N^{+}, j \in N^{+}, z \in M \\
\sum_{j=1}^{n} c_{0 j z} \geq 1, & \forall z \in M \\
\sum_{i=1}^{n} c_{i(n+1) z} \geq 1, & \forall z \in M \\
\sum_{j=1}^{n+1} c_{i j z} \leq 1, & \forall i \in N, z \in M
\end{array}
$$




$$
c_{i i z}=0, \quad \forall i \in N^{+}, z \in M
$$

where (3)-(4) guarantee that for each information type there is at least one communication link from a source to a node and there must be at least one communication link between a node and the base station, respectively. Note that constraint (3) defines an initial state of the network flow at each decision time interval. Constraint [5] enforces that there is only one communication link of each data type out of a node. Constraint (6) prevents self communication.

Let $\lambda_{i j z} \geq 0$ denote the average rate (packets per second) at which data of type $z$ is transmitted from node $i$ to node $j$. Note that $\lambda_{0 j z}$ represents the sensing rate of data type $z$, assumed to be a constant equal to $\bar{\lambda}_{z}$ packets per time interval. Following the definition of the communication link matrix $C$, $\lambda_{i j z}$ needs to satisfy:

$$
\begin{aligned}
& \lambda_{i j z}=0 \Rightarrow c_{i j z}=0, \forall i \in N^{+}, j \in N^{+}, z \in M, \\
& \lambda_{i j z}>0 \Rightarrow c_{i j z}=1, \forall i \in N^{+}, j \in N^{+}, z \in M .
\end{aligned}
$$

Constraint (7) says that if there is data flow between two nodes, then the link assignment should be active. The constraint (7) can be implemented as the following inequality constraints:

$$
\epsilon c_{i j z} \leq \lambda_{i j z} \leq\left|G_{z}\right| \bar{\lambda}_{z} c_{i j z}, \forall i \in N^{+}, j \in N^{+}, z \in M,
$$

where $\epsilon$ is a sufficiently small positive number and $\left|G_{z}\right|$ is the total number of sensors of data type $z$. In other words, suppose $\lambda_{i j z} \neq 0$, then $(8)$ is true if and only if $c_{i j z}=1$. Suppose $\lambda_{i j z}=0$, then $(8)$ is true if and only if $c_{i j z}=0$.

Denote $a_{i z} \in\{0,1\}, \forall i \in N, z \in M$ as the data type aggregator assignment, where by definition

$$
a_{i z}=1 \Longleftrightarrow \sum_{j=0}^{n} c_{j i z}>1, \forall i \in N, z \in M .
$$

In other words, if there are more than one packets of the same data type transmitted to a node, then the node will act as an aggregator. Constraint (9) can be written as a set of linear inequalities as follows:

$$
\begin{array}{ll}
(1-n) a_{i z}+\sum_{j=0}^{n} c_{j i z} \leq 1, & \forall i \in N, z \in M, \\
(1+\epsilon) a_{i z}-\sum_{j=0}^{n} c_{j i z} \leq 0, & \forall i \in N, z \in M,
\end{array}
$$

where $\epsilon$ is a sufficiently small positive number. To guarantee a feasible communication link, the data flow within the node needs to be conserved, i.e. the incoming data equals the aggregated outgoing data:

$$
\begin{aligned}
& \sum_{z=1}^{m} \sum_{j=1}^{n+1} c_{i j z} \lambda_{i j z}=\sum_{z=1}^{m} \sum_{j=0}^{n} c_{j i z} \lambda_{j i z}\left(1+\left(\gamma_{z}-1\right) a_{i z}\right), \\
& \forall i \in N, z \in M,
\end{aligned}
$$

where $0 \leq \gamma_{z} \leq 1$ is the aggregation ratio of data type $z$. Observe that when $\gamma_{z}=1$, then there is no data aggregation/processing.

Since the nodes are communicating via wireless network, the channel bandwidth are shared among the nodes. This

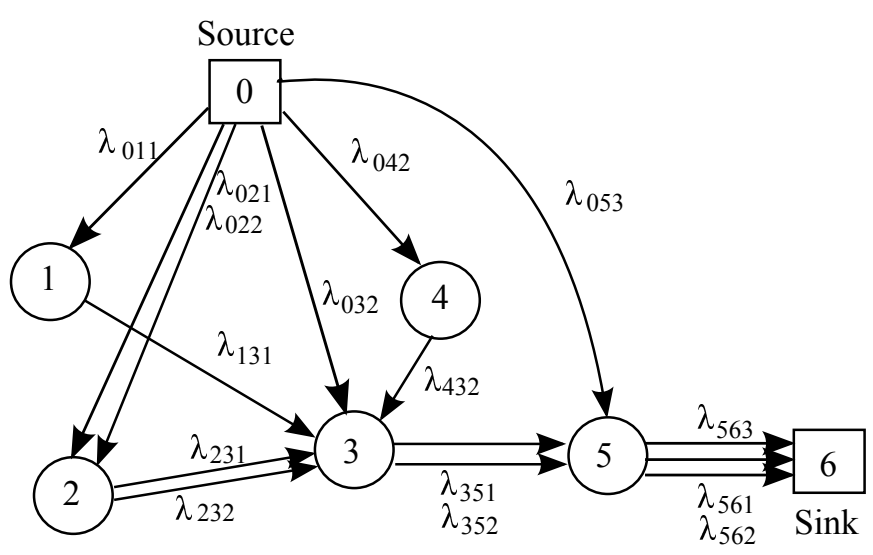

Fig. 3: Aggregation Network Topology Example

implies that communication between two nodes restrains available bandwidth to other neighbor nodes. Therefore, bandwidth limitation should be considered in our formulation as well, i.e. all communication data (number of transmitting/receiving bits) should not be greater than the channel bandwidth limitation. Specifically, the bandwidth constraints can be formulated as

$$
\sum_{z=1}^{m} \sum_{j=1}^{n+1} c_{i j z} \lambda_{i j z} L+\sum_{z=1}^{m} \sum_{j=1}^{n} c_{j i z} \lambda_{j i z} L \leq B h, \forall i \in N,
$$

where $B$ is the channel bandwidth (bits per second), $h$ is the decision time interval and $L$ is the packet length (number of bits per packet).

Finally, we will use an example scenario to show the information flow topology that can be achieved from our model. Consider Figure 3 where the system is composed of five UAVs that are given a mission to retrieve three different types of information. Nodes 1, 2 and 4 are sensor nodes, node 3 is both a sensor and an aggregator, while node 5 is a sensor as well as a relay node. The correlated data obtained from node $1\left(\lambda_{131}\right)$ and node $2\left(\lambda_{231}\right)$ are processed within node 3 . At the same time, the data obtained from nodes $2\left(\lambda_{232}\right), 3\left(\lambda_{032}\right)$ and $4\left(\lambda_{432}\right)$ are also processed within node 3 . Specifically, from (11), the outgoing data flow after the aggregation within node 3: $\lambda_{351}=\left(\lambda_{131}+\lambda_{231}\right) \gamma$ and $\lambda_{352}=\left(\lambda_{032}+\lambda_{232}+\lambda_{432}\right) \gamma$. Both processed data streams/packets are relayed to node 5, which are transmitted to the base station. Note that node 5 acts as a relay node because the data received from node 3 and its own data are of different types.

\section{B. Energy Models}

We will adopt an energy consumption model, which has been commonly used in the wireless sensor network literature [54]-[56]. The total energy in most multi-UAV applications is composed of three terms. The first term is the sensing energy $E^{s}$, which is the energy used to sense a target/AOI. We will assume that the energy to sense one bit of information is a constant equal to $\epsilon_{s} \mathrm{~J}$. The sensing energy consumed by node $i$ within the time interval is

$$
E_{i}^{s}\left(c_{0 i z}\right):=\epsilon_{s} L \sum_{z=1}^{m} \bar{\lambda}_{z} c_{0 i z}, \forall i \in N \text {. }
$$


The second one is the aggregation energy $E^{p}$, which is the energy to do data processing. The energy to process one bit of information is also assumed to be a constant equal to $\epsilon_{p} \mathrm{~J}$. The aggregation energy consumed by node $i$ within the time interval is

$$
\begin{aligned}
E_{i}^{p}\left(c_{j i z}, \lambda_{j i z}, a_{i z}\right):= & \epsilon_{p} L \sum_{z=1}^{m} \bar{\lambda}_{z} c_{0 i z} a_{i z}+ \\
& \epsilon_{p} L \sum_{z=1}^{m} \sum_{j=1}^{n} c_{j i z} \lambda_{j i z} a_{i z}, \forall i \in N .
\end{aligned}
$$

The last energy term is the communication cost, which is composed of two parts: the transmitting energy $E^{t}$ and the receiving energy $E^{r}$. The transmitting energy depends on the distance between the nodes $d_{i j}$, i.e. $E^{t}\left(d_{i j}\right):=\epsilon_{t}+$ $\epsilon_{r f} d_{i j}^{\beta}$, where $\beta \geq 2$ is the path loss exponent, $\epsilon_{t}(\mathrm{~J} / \mathrm{bit})$ and $\epsilon_{r f}\left(\mathrm{~J} / \mathrm{bit} / \mathrm{m}^{\beta}\right)$ are constants. The energy of receiving one bit of information is assumed to be a constant equal to $\epsilon_{r}$ $\mathrm{J}$. The receiving energy consumed by node $i$ within the time interval is

$$
E_{i}^{r}\left(c_{j i z}, \lambda_{j i z}\right):=\epsilon_{r} L \sum_{z=1}^{m} \sum_{j=1}^{n} c_{j i z} \lambda_{j i z}, \forall i \in N .
$$

The transmitting energy consumed by node $i$ within the time interval is

$E_{i}^{t}\left(c_{i j z}, \lambda_{i j z}, d_{i j}\right):=\sum_{z=1}^{m} \sum_{j=1}^{n+1}\left(\epsilon_{t}+\epsilon_{r f} d_{i j}^{\beta}\right) c_{i j z} \lambda_{i j z} L, \forall i \in N$.

The total energy used by node $i$ for sensing a target/AOI, processing information and communication during the time interval is denoted by

$$
E_{i}:=E_{i}^{s}+E_{i}^{p}+E_{i}^{r}+E_{i}^{t}, \forall i \in N .
$$

Let $e_{i}$ be the energy stored in the $i^{\text {th }} \mathrm{UAV}$ at time $t$, then the remaining energy $e_{i}^{+}$at time $t+h$ is given by

$$
e_{i}^{+}:=e_{i}-E_{i} \geq 0, \forall i \in N \text {. }
$$

\section{UAV Dynamic Constraints}

The two-dimensional UAV kinetic model is given by:

$$
\left[\begin{array}{c}
\dot{x}_{i} \\
\dot{y}_{i}
\end{array}\right]=f\left(\varphi_{i}, v_{i}, \psi_{i}\right)=\left[\begin{array}{c}
v_{i} \cos \psi_{i} \\
v_{i} \sin \psi_{i}
\end{array}\right], \forall i \in N
$$

where $\varphi_{i}=\left[x_{i} y_{i}\right]^{T}$ is the inertial position, $v_{i}$ is the speed and $\psi_{i}$ is the heading of the $i^{t h}$ UAV. We will assume that UAVs fly at a constant speed and heading in the interval $[t, t+h]$ and are subject to the following constraint:

$$
v_{\min } \leq v_{i} \leq v_{\max }, \forall i \in N,
$$

where $v_{\min }$ and $v_{\max }$ are lower and upper bounds on speed.

Moreover, since we assume that the UAVs are in one-hop communication range and to avoid collision among UAVs at each time interval, the following constraints are necessary:

$$
r_{c}>d_{i j} \geq r_{\text {safe }}, \forall i \neq j,(i, j) \in N \times N,
$$

where $r_{c}$ is a sufficiently large positive number defined as a communication range limit, $d_{i j}$ is the distance between two nodes and $r_{\text {safe }}$ is the safety distance.

\section{State Update Equation}

Let $k$ denote the $k^{\text {th }}$ decision making round at time interval $\left[t_{k}, t_{k+1}\right]$, i.e. $t_{k+1}-t_{k}=h$. The state $X_{i}$ and the control input $u_{i}$ for the $i^{t h} \mathrm{UAV}$ are defined as

$$
\begin{array}{ll}
X_{i}:=\left(e_{i}, \varphi_{i}\right), & \forall i \in N, \\
u_{i j z}:=\left(c_{0 i z}, c_{i j z}, \lambda_{i j z}, a_{i z}, v_{i}, \psi_{i}\right), & \forall i \in N, z \in M, \\
& j \in N \cup\{n+1\},
\end{array}
$$

where $X:=\left(X_{1}, X_{2}, \ldots, X_{n}\right)$ is the state of the overall system. The components of the overall system control input $u$ are all $u_{i j z}, i \in N, j \in N \cup\{n+1\}, z \in M$.

Obviously, all the variables in the previous sections can be considered as a function of $k$. Let $X(k)$ denote the state of the overall system and $u(k)$ denote the system control input at time $t_{k}$. The overall system state update equation is given by

$$
X(k+1)=\phi(X(k), u(k), k), \forall k,
$$

where $\phi$ can be derived from (18) and (19).

\section{Optimal Control Problem}

We formulate the optimal control problem to determine the roles of the UAVs as an MINLP. We apply this formulation to a multi-UAV target tracking application and a multi-UAV mapping application. The MINLP is solved at each time instant $t_{k}$.

I) Target Tracking: Though our main objective is to minimize the total energy consumed by all nodes in the system (17), for the target tracking application the target tracking accuracy should be considered as well. Particularly, a shorter distance between the sensor and the target will result in a higher contribution to the tracking accuracy. Therefore, it is more energy-efficient to select only a subset of sensors that are reasonably close to the target for tracking. This objective can be incorporated as a constraint that guarantees a minimum information contribution $\pi_{\min }$ requirement as

$$
\pi:=\sum_{z=1}^{m} \sum_{i=1}^{n} c_{0 i z} \operatorname{tr}\left\{H_{i}(t)^{T} \log \left(R_{i}^{-1}(t)\right) H_{i}(t)\right\} \geq \pi_{\min }
$$

where $\pi$ is the information contribution, $H_{i}(t)$ is the observation model and $R_{i}(t)$ is the measurement noise covariance. Note that $H_{i}(t)^{T} \log \left(R_{i}^{-1}(t)\right) H_{i}(t)$ represents the information contribution of sensor $i$ to the tracking error of the target at time $t$. Furthermore, our definition of information contribution is slightly different from the one defined and used in [35][37]. Specifically, we took the natural logarithm of the inverse of $R_{i}(t)$ to reduce the decay rate of information contribution in order to match with the target tracking application using mobile agents, i.e. the useful information can be obtained within a reasonable distance between the sensor and the target.

The sensing range limit can be implemented as the following constraint:

$$
c_{0 j z}\left(d_{0 j}^{2}-r_{s}^{2}\right) \leq 0, \forall j \in N, z \in M,
$$

where $d_{0 j}$ is the distance between the node and the target and $r_{s}$ is the maximum sensing range. Constraint 26 states that 
if a node is a sensor, then the distance between the node and the target has to be less than the maximum sensing range. Note that the square of the distance is chosen for an easier implementation.

The multi-UAV target tracking problem can be formulated as the following optimal control problem: Given $n$ UAVs, a target and a base station, determine a role for each UAV, a communication network link and a UAV trajectory that solves

$\begin{array}{ll}\underset{u}{\operatorname{minimize}} & \sum_{i=1}^{n} E_{i} \\ \text { subject to } & \text { (2) }-(6),(8),(10)-21, \\ & \text { (25) and }\end{array}$

II) Area Mapping: Given $n$ UAVs, an AOI, a base station and a UAV trajectory, determine a role for each UAV and a communication network link that solves

$$
\begin{array}{cl}
\underset{u}{\operatorname{minimize}} & \sum_{i=1}^{n} E_{i} \\
\text { subject to } & \text { (2)-(6), (10)-(18) and } \\
& v_{i}=V_{i}, \forall i \in N, \\
& \psi_{i}=\Psi_{i}^{d}, \forall i \in N, \\
& c_{0 i z}=C_{i}, \forall i \in N,
\end{array}
$$

where $V_{i}$ is the constant speed of the vehicle and $\Psi_{i}^{d}$ is the desired heading angle of the path. $C_{i}$ is a pre-determined data type sensor assignment vector. Also, note that for an area surveying/mapping application, the UAV dynamic constraints described in Section III-C are not included because we assume that the trajectory of each UAV and the collision avoidance among UAVs are decided by a path planning controller.

\section{Applications}

This section provides simulation results to illustrate the correctness and effectiveness of our framework in trading off communication and computation energy consumption in multiUAV applications. A multiple UAV single-target tracking and area mapping application are chosen as our demonstration examples. All simulations were simulated on MATLAB [57] and the MINLP was modelled using OPTI TOOLBOX [58] and solved with SCIP [59]. We carry out a single simulation to compute the results. We do not carry out Monte-Carlo simulations as there are no random parameters in the simulation settings and hence the results are similar for any number of simulations.

\section{A. Target Tracking}

1) Target and Sensor Models: For a target tracking application, we will follow the work of [37] to set up the optimization problem to make a decision on a subset of the UAVs to be sensor nodes. The motion of a target will be modelled as a linear discrete-time Markov process:

$$
X_{0}(t+1)=F_{0}(t) X_{0}(t)+w_{0}(t) \text {, }
$$

where $X_{0}(t)$ is the state vector of a target, $F_{0}(t)$ is the state transition matrix and $w_{0}(t)$ is the process noise assumed to be zero mean Gaussian noise with covariance $Q_{0}(t)$.

The measurement equation of a sensor is

$$
Z_{i}(t)=H_{i}(t) X_{0}(t)+\nu_{i}(t),
$$

where $\nu_{i}(t)$ is the measurement noise assumed to be zero mean Gaussian with covariance $R_{i}(t)$. We will assume that the measurement noise covariance is a function of the distance between a sensor and a target, i.e. $R_{i}(t):=K(t) d_{0 i}^{\beta}(t)$, where $K(t)$ is a distance-independent coefficient, and $d_{0 i}(t)$ is the distance from a sensor to a target. Moreover, we will also assume that the measurement noise covariances are uncorrelated between any two nodes.

2) Information Filter: For multi-sensor data fusion, we use an information filter [37], [60], which is an inverse covariance form of the Kalman filter. Let $\hat{X}_{0}(t \mid t)$ and $\hat{X}_{0}(t+1 \mid t)$ denote the target estimated state vector and target predicted state vector, respectively. Define the information matrix $Q(t \mid t):=$ $P^{-1}(t \mid t)$ and $Q(t+1 \mid t):=P^{-1}(t+1 \mid t)$, the information state vector $\hat{q}(t \mid t):=P^{-1}(t \mid t) \hat{X}_{0}(t \mid t)$ and $\hat{q}(t+1 \mid t):=$ $P^{-1}(t+1 \mid t) \hat{X}_{0}(t+1 \mid t)$, where $P(t \mid t)$ and $P(t+1 \mid t)$ are the covariances of the estimation error $X_{0}(t \mid t)-\hat{X}_{0}(t \mid t)$ and the prediction error $X_{0}(t+1 \mid t)-\hat{X}_{0}(t+1 \mid t)$. The prediction and estimation steps are

Estimation:

$\hat{q}(t \mid t)=\hat{q}(t \mid t-1)+H_{i}^{T}(t) R_{i}^{-1}(t) Z_{i}(t)$,

$Q(t \mid t)=Q(t \mid t-1)+H_{i}^{T}(t) R_{i}^{-1}(t) H_{i}(t)$,

Prediction:

$\hat{q}(t+1 \mid t)=Q(t+1 \mid t) F_{0}(t+1) Q^{-1}(t \mid t) \hat{q}(t \mid t)$,

$Q(t+1 \mid t)=\left(F_{0}(t+1) Q^{-1}(t \mid t) F_{0}^{T}(t+1)+Q_{0}(t+1)\right)^{-1}$.

For multi-sensor data fusion, i.e more than one node tracking the target, (30) and 31) are replaced, respectively by

$$
\begin{aligned}
& \hat{q}(t \mid t)=\hat{q}(t \mid t-1)+\sum_{i \in S} H_{i}^{T}(t) R_{i}^{-1}(t) Z_{i}(t), \\
& Q(t \mid t)=Q(t \mid t-1)+\sum_{i \in S} H_{i}^{T}(t) R_{i}^{-1}(t) H_{i}(t),
\end{aligned}
$$

where $S$ is a set of sensor nodes.

3) Simulation settings: For simplicity, we consider a small UAV network, i.e. $n=3$, which are deployed to track a single target in a two-dimensional area and needs to periodically report the target state back to the base station. Note that we consider the single target state as one data type. The base station is at $(0,0)$. The initial positions of the UAVs are at positions $(0,100),(100,0)$, and $(100,100)$. The target initial position is $(20,20)$. The target state vector $X_{0}(t)$ in 28) is composed of the target positions in the $x$ and $y$ axes, and velocities in the $x$ and $y$ axes, denoted as $v_{x}$ and $v_{y}$, respectively. The parameters corresponding to the target state (28), measurement equations 29] and information filter are [37]:

$F_{0}(t)=\left(\begin{array}{cccc}1 & 0 & 1 & 0 \\ 0 & 1 & 0 & 1 \\ 0 & 0 & 1 & 0 \\ 0 & 0 & 0 & 1\end{array}\right), Q_{0}(t)=\left(\begin{array}{cccc}2 & 0 & 0 & 0 \\ 0 & 2 & 0 & 0 \\ 0 & 0 & 0.04 & 0 \\ 0 & 0 & 0 & 0.04\end{array}\right), \forall t$ 


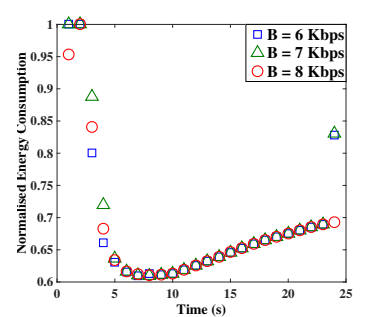

(a)

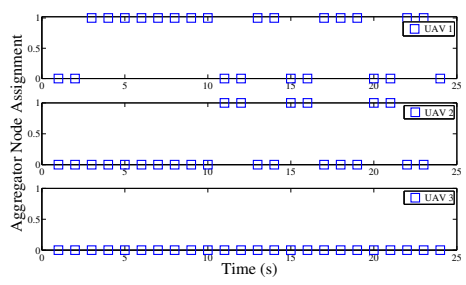

(b)
Fig. 4: (a) Normalised total energy consumption for different channel bandwidths with respect to baseline scheme (b) Aggregator node assignments at different time steps for channel bandwidth $B=7 \mathrm{Kbps}$.

$$
\begin{aligned}
& H_{i}(t)=\left(\begin{array}{llll}
1 & 0 & 0 & 0 \\
0 & 1 & 0 & 0
\end{array}\right), K(t)=\left(\begin{array}{cc}
1 \times 10^{-6} & 0 \\
0 & 1 \times 10^{-6}
\end{array}\right), \\
& \hat{q}(1 \mid 0)=\left(\begin{array}{c}
0 \\
0 \\
0 \\
0
\end{array}\right), Q(1 \mid 0)=\left(\begin{array}{cccc}
1 & 0 & 0 & 0 \\
0 & 1 & 0 & 0 \\
0 & 0 & 1 & 0 \\
0 & 0 & 0 & 1 .
\end{array}\right)
\end{aligned}
$$

For all simulations, we let the target velocities be $v_{x}=$ $10 \mathrm{~m} / \mathrm{s} v_{y}=15 \mathrm{~m} / \mathrm{s}$. The UAV parameters [61] are $v_{\min }=$ $10 \mathrm{~m} / \mathrm{s}, v_{\max }=30 \mathrm{~m} / \mathrm{s}$, the initial UAV energy budget is $100 \mathrm{KJ}$, the communication range $r_{c}=500 \mathrm{~m}$, the sensing range $r_{s}=200 \mathrm{~m}$, the safety distance $r_{\text {safe }}=50 \mathrm{~m}$, the decision time interval $h$ is $1 \mathrm{~s}$. The energy parameters [14] are $\epsilon_{s}=50 \mathrm{~nJ} / \mathrm{bit}, \epsilon_{p}=10 \mathrm{~nJ} / \mathrm{bit}, \epsilon_{r}=135 \mathrm{~nJ} / \mathrm{bit}, \epsilon_{t}=$ $45 \mathrm{~nJ} / \mathrm{bit}, \epsilon_{r f}=0.1 \mathrm{~nJ} / \mathrm{bit} / \mathrm{m}^{2}, \quad \gamma_{z}=0.7, \beta=2, L=1024$ bits/packet, $\bar{\lambda}_{z}=5$ packets/time interval and $\pi_{\min }=6$.

4) Simulation Results: We compare the results obtained from the MINLP with a baseline strategy where all sensor nodes individually communicate with the base station using a single-hop communication protocol. The comparison is performed in terms of energy consumed per decision time interval $[t, t+h]$ between the MINLP and the baseline strategy. The vertical axis in Figure 4a represents the system energy consumption per decision time $[t, t+h]$ normalized by the baseline scheme. Our simulation suggests that the channel bandwidth constraint has an effect on the energy consumption of the system due to the restriction on the information flow pattern. Specifically, when the bandwidth is limited below the threshold value of $5 \mathrm{Kbps}$ (not shown on the plot), the MINLP algorithm cannot find a solution that is better than the baseline strategy, hence no energy saving can be obtained. However, when the channel bandwidth is above the threshold, the MINLP can provide an optimal strategy that can save energy consumption up to $40 \%$ compared to the baseline strategy, as shown in Figure $4 \mathrm{a}$. However, the energy saving improvement cannot be observed with an increase in $B>6 \mathrm{Kbps}$. Figure $4 \mathrm{~b}$ shows the aggregator role assignments of each UAV at each time instance of the simulation, where 1 refers to an active role.

\section{B. Area mapping}

A team of $n$ UAVs are deployed to survey a rectangular region with a length of $T$ meters and a width of $W$ meters using cameras. The vehicles are subject to communication, sensing and energy constraints. Each UAV has a sensing range of $r_{s}$ meters determined by the camera resolution and altitude. Typically, mapping applications are performed using a lawnmowing pattern and hence we split the rectangular region into lanes of width $\zeta r_{s}$, where $0<\zeta \leq 1$ is the overlap factor. $\zeta=$ 1 implies the distance between the lanes is $r_{s}$ and there is no overlap of sensing regions between the aerial survey of UAVs, while $0<\zeta<1$ implies there is some overlap of the sensor footprint between two adjacent lanes. In terms of area coverage $\zeta=1$ is the best strategy. However, for mapping purposes, there must be at least $50 \%$ overlap between two lanes to create good mosaics [62]. We assume a linear relationship between the overlap factor and the data aggregation ratio, i.e. $\zeta=\gamma$, which means that the higher the overlapping area, the higher $t$ the data reduction after data processing. Note that here we assume that the overlap factor is a constant and the same for all nodes, therefore the subscript $z$ of $\gamma$ notation is dropped. The number of lanes are $N_{\ell}:=\left\lceil\frac{T}{2 \zeta r_{s}}\right\rceil+1$ and each lane is denoted by $\ell_{\kappa}, \kappa=1, \ldots, N_{\ell}$. The vehicles use waypoint navigation for the survey and hence each lane $\ell_{\kappa}$ is represented by two waypoints $\ell_{\kappa}=\left(\omega_{\kappa}^{b}, \omega_{\kappa}^{t}\right)$, where, $\omega_{\kappa}^{b}=\left(x_{\kappa}^{b}, y_{\kappa}^{b}\right), \omega_{\kappa}^{t}=$ $\left(x_{\kappa}^{t}, y_{\kappa}^{t}\right)$ as shown in Figure 5a. Lane $\ell_{\kappa}$ can be accurately tracked using any accurate path following algorithm [63].

The time taken by the UAV team to survey the complete region depends on the number of UAVs deployed; when $n=$ 1 , the lower bound on the mission time is $W T N_{\ell}$ seconds. Initially, UAV $i$ is given a lane $\ell_{i}, i \in N$ in terms of their waypoints $\ell_{i}=\left(\omega_{i}^{b}, \omega_{i}^{t}\right)$. Once the vehicle reaches $\omega_{i}^{t}$, the lane $\ell_{i+n}=\left(\omega_{i+n}^{t}, \omega_{i+n}^{b}\right)$ is assigned. However, we can see that UAV $i$ is assigned the waypoint sequence $\left(\omega_{i}^{b}, \omega_{i}^{t}\right)$ for the first lane while $\left(\omega_{i+n}^{t}, \omega_{i+n}^{b}\right)$ was assigned the next lane. If we assigned $\left(\omega_{i+n}^{b}, \omega_{i+n}^{t}\right)$, then the vehicle has to travel from $\omega_{i}^{t}$ to $\omega_{i+n}^{b}$, which is unproductive travel, since the vehicle expends fuel without surveying any of the region. Hence, we assign the UAV with an alternating sequence of waypoints.

The desired heading angle $\psi_{i}^{d}$ is determined as

$$
\psi_{i}^{d}= \begin{cases}\arctan \left(y_{\kappa}^{b}-y_{\kappa}^{t}, x_{\kappa}^{t}-x_{\kappa}^{b}\right) & \text { if } \ell_{\kappa}=\left(\omega_{i}^{b}, \omega_{i}^{t}\right) \\ \arctan \left(y_{\kappa}^{b}-y_{\kappa}^{t}, x_{\kappa}^{b}-x_{\kappa}^{t}\right) & \text { if } \ell_{\kappa}=\left(\omega_{i}^{t}, \omega_{i}^{b}\right) .\end{cases}
$$

1) Simulation Setting: We consider a region of $3000 \mathrm{~m} \times 3000 \mathrm{~m}$ and the base station is located in the middle at $(1500,1500)$. The sensing range of the vehicles $r_{s}=100 \mathrm{~m}$, the communication range $r_{c}=2500 \mathrm{~m}$ and the speed of the vehicles is $10 \mathrm{~m} / \mathrm{s}$. We assume three vehicles are deployed to perform the mapping. The parameters used in the simulation are $\epsilon_{s}=50 \mathrm{~nJ} / \mathrm{bit}, \epsilon_{p}=10 \mathrm{~nJ} / \mathrm{bit}, \epsilon_{r}=$ $135 \mathrm{~nJ} / \mathrm{bit}, \epsilon_{t}=45 \mathrm{~nJ} / \mathrm{bit}, \epsilon_{r f}=0.1 \mathrm{~nJ} / \mathrm{bit} / \mathrm{m}^{2}, \beta=2$, $L=1280 \times 720$ bits/packet and $\bar{\lambda}_{z}=5$ packets/time interval. Each UAV communicates to the base station every $h=5$ seconds. The vector field based path following algorithm [64] is selected as the UAV path planning controller. The vector field based path following approach uses a two-fold strategy. When the vehicle is far away from the desired path, the algorithm directs the vehicle towards the path until the vehicle is $\tau$ meters from the path as shown in Figure 5b, where the parameter $\tau$ is the transition boundary between moving towards the path and following the path. The vehicle then 


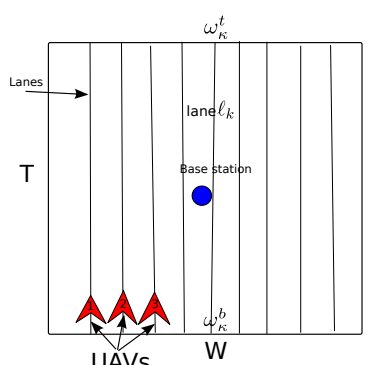

(a)

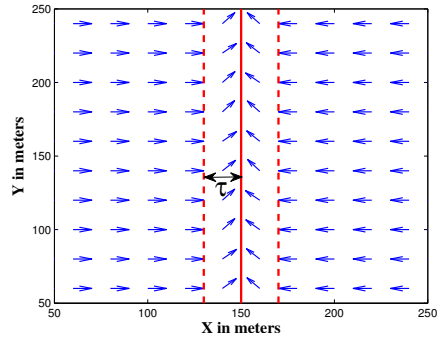

(b)
Fig. 5: (a) The search area is decomposed into lanes and each $\mathrm{UAV}$ is assigned to one lane. Once the UAV completes one lane, then another lane is assigned. (b) The vector field of the vehicle at various locations is shown. $\tau=20$ and $\chi=\pi / 3$.

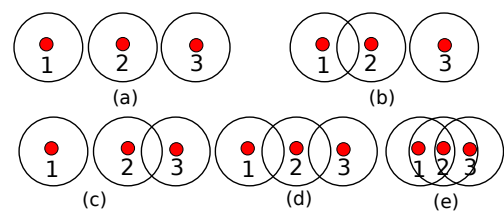

Fig. 6: (a) No common data between the nodes (b) nodes 2 and 3 have common data of type 1 (c) node 2 and node 1 has common data of type 0 (d) node 2 has common data of type 0 with node 1 and type 1 with node 3 (e) all the nodes have common data of type 0 and 1 .

transits into following the desired path with an entry angle of $\chi$. The effects of $\tau$ and $\chi$ are well studied in [64] and [25]. For all simulations, we use $\tau=20$ meters and $\chi=\pi / 3 \mathrm{rad}$.

For the mapping application, the values for $c_{0 i z}$ depends on the distance between the nodes. That is, if the distance is greater than twice that of the sensing range $r_{s}$, then we will assume that the sensing data are not related and cannot be aggregated. In other words, the data are of different types. In order to illustrate how $c_{0 i z}$ values are determined at each decision interval, consider a three vehicle system in Figure 6 a) where a distance between node $i$ and node $j d_{i j}>2 r_{s}$. For this scenario, there is no common data type between the nodes due to no overlap of the sensed regions, i.e. $z \in\{0,1,2\}$. Therefore, the values of $c_{010}=1, c_{011}=0, c_{012}=0, c_{020}=$ $0, c_{021}=1, c_{022}=0, c_{030}=0, c_{031}=0$ and $c_{032}=1$, which implies that none of the nodes have common data type.

Now consider the scenario as shown in Figure 6(b), where nodes 1 and 2 have a common data type $z=0$ and node 3 is distant from nodes 1 and 2. Therefore, in this case, we have $c_{010}=1, c_{011}=0, c_{020}=1, c_{021}=0, c_{030}=0$ and $c_{031}=1$. Similarly, we can determine values for other agent configurations shown in Figure 6

2) Simulation Results: The bandwidth allocated to communicate with the base station plays a key role in determining the computing nodes. Figure 7 shows the total energy consumption of the MINLP normalised to the baseline strategy for every $h=5$ seconds with an overlap factor $\zeta=0.5$. When the available bandwidth is less than $6 \mathrm{Mbps}$ (not shown on the plot), the nodes communicate directly to the base station. Hence, we do not show this effect. However, when we increase

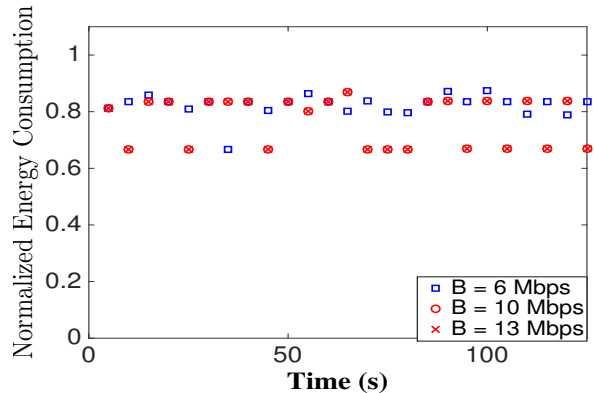

Fig. 7: The normalized total energy of the MINLP compared to the baseline strategy for different bandwidth constraints having $\zeta=0.5$.

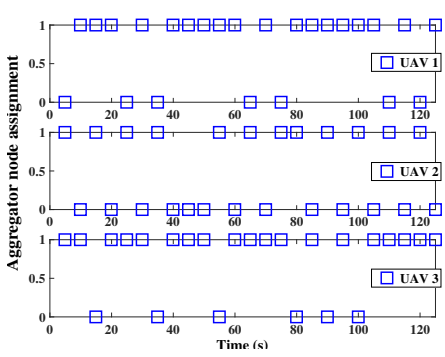

(a) $\mathrm{B}=6 \mathrm{Mbps}$

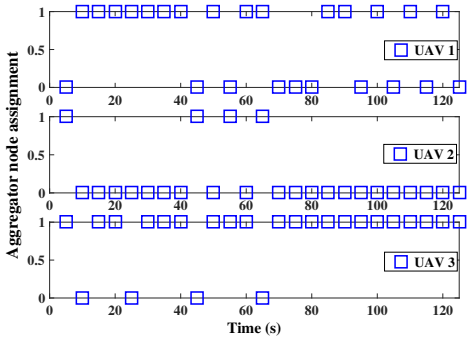

(b) $\mathrm{B}=10 \mathrm{Mbps}$
Fig. 8: The aggregator node selection at different time steps when the bandwidth parameter is varied for the same overlap $\zeta=0.5$. (a) $\mathrm{B}=6 \mathrm{Mbps}$ and (b) $\mathrm{B}=10 \mathrm{Mbps}$.

the bandwidth, data aggregation behaviours can be observed. As shown in Figure 7) the energy saving is close to $20 \%$ for most of the decision cycles (for $B=6 \mathrm{Mbps}$ ). With further increase in bandwidth to $B=10 \mathrm{Mbps}$, we can see that there is further increase in energy saving of $35 \%$. However, with additional increase in bandwidth to $B=13 \mathrm{Mbps}$, there is no further improvement in energy saving. As expected, the energy reduction is due to co-operation among the agents, i.e. when the bandwidth is sufficiently large, more energyefficient feasible information flow patterns are allowed.

Effect of overlap factor: In the mapping application, the overlap factor $\zeta$ plays a key role in determining the amount of information that needs to be transmitted by the aggregator node to the base station. When $\zeta$ increases, the agents are close to each other with high overlap. Therefore, during the mosaic operation, the resultant image size will be smaller compared to the sum of individual images. In order to validate this hypothesis, we carried out experiments with different overlap factors $\zeta=0.3,0.5,0.7$ and 0.9 for the same bandwidth of 10 Mbps. In Figure 9a, we can see the effect of $\zeta$ for a given bandwidth. Specifically, the energy saving increases as $\zeta$ increases. For example, when $\zeta=0.9$, we can achieve savings up to $60 \%$ compared to the baseline strategy.

We further, carry out simulations for 5 agents having the same simulation parameters as above. Figure $9 \mathrm{~b}$ shows the respective energy saving when 5 agents perform the survey. With increasing overlap factor, the amount of information to be dispatched reduces and hence there is a decrease in energy 


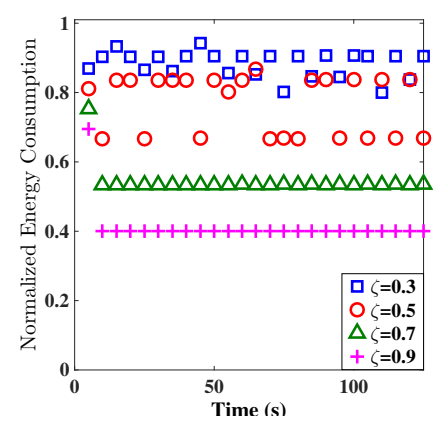

(a)

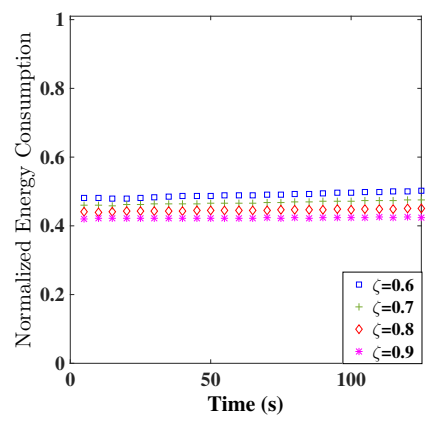

(b)
Fig. 9: The normalized total energy of the MINLP with reference to the baseline strategy for different overlap factors. (a) For 3 agents with channel bandwidth of $B=10 \mathrm{Mbps}$. (b) For 5 agents with channel bandwidth of $B=20 \mathrm{Mbps}$.

consumption. With increase in number of agents we can see that a trend in energy conversation similar to that of agent 3 simulation can be seen.

Energy savings: For the given simulation setting in the mapping application, the total energy consumed by the baseline strategy for 3 agents is 524030 Joules while that of the MINLP is 335310 Joules. The MINLP strategy gives a saving of 188720 Joules, which results in 95 seconds of additional flight time, including communicating with the base station 19 times. The speed of the UAV is $10 \mathrm{~m} / \mathrm{s}$ and hence the additional distance travelled is $950 \mathrm{~m}$. Similarly for the 5 agents case, the baseline strategy consumes 743680 Joules while our proposed method consumes 405130 Joules. This gives a saving of 338550 Joules that results in 135 seconds of additional flight time for each agent and the agents can communicate to the base station 27 times. Since the speed of the vehicle is $10 \mathrm{~m} / \mathrm{s}$, the additional distance the vehicles can travel using the proposed approach is $1350 \mathrm{~m}$.

Comparison with rigid structures: We compare the performance of the MINLP approach against some representative rigid hierarchical structures and baseline strategy. Since there are multiple agents, a rigid hierarchical structure represents one type of solution that may not be optimal. For a 3 agent scenario, we consider two hierarchical rigid structures - H1 and $\mathrm{H} 2$, as shown in Figure 10(a). In H1 structure, vehicles 1 and 3 broadcast their information to agent 2 which in turn assimilates the information and broadcasts to the base station. Another type of rigid structure is $\mathrm{H} 2$, where agents 1 and 2 broadcast the information to agent 3 which assimilates the information and then broadcasts to the base station. As the agents are deployed in lanes (see Figure 5a), the distance between agent 1 and the base station is greater than the distance between agent 3 and the base station, and hence we did not consider a rigid structure where only agent 1 is communicating. Figure 10 (b) shows the normalized mission time for each of these strategies. The mission time represents the time to accomplish the coverage until one of the agent's drains its battery. We normalize by taking the time taken by the baseline strategy as the reference.
The performance of the rigid structure is poor, because the agents that need to communicate with the base station is fixed. Therefore, the agents that communicate with the base station will drain their battery quicker than the non-base station communicating agents. The strategy $\mathrm{H} 2$ performs better than $\mathrm{H} 1$ since the communicating agent is closer to the base station in $\mathrm{H} 2$ than in $\mathrm{H} 1$ strategy. The MINLP strategy performs the best.

Similarly, we carried out a comparison between rigid structures, baseline strategy and the proposed strategy for 5 agents. With 5 agents, there can be different combinations in which clusters can be formed. For example, clusters can be: $\{1,2,3,4,5\}$ - individual agents, or $\{(1,2), 3,4,5\}-$ a set of two agents cooperating while the others transmitting directly to the base station or $\{(1,2,3), 4,5\}$ - a team of three agents and the rest directly communicate to the base station or $\{(1,2)(3,4), 5\}$ - two agents teams of different combinations or $\{(1,2,3,4), 5\}$ - four agent teams with a single member broadcasting to the base station. It is difficult to compare with all of the combinations in which rigid structure can be formed and hence we select few representative ones as shown in Figure 10 (c). The strategy $\mathrm{H} 3$ and $\mathrm{H} 4$ represents a scenario where the agent 3 and agent 5 are the information assimilation as well as broadcast agents to the base station respectively. The strategy $\mathrm{H} 5$ represents a case where there are two data assimilation and broadcasting agents to the base station - agent 2 and 4 . Even for 5 agents, the rigid structures have lowest performance compared to the baseline strategy as shown in Figure 10(d), while the MINLP performs the best.

Effect of increasing the number of agents: We carried out empirical evaluation through simulation by increasing the number of agents and recording the average time taken to compute the solution. It is well known that the MINLP computational complexity increases with an increase in the number of agents which is also depicted in the Figure 11 Our formulation works well till 10 number of agents, for which the average time taken is 2.67 seconds. Therefore, in order to reduce the computational complexity one may have to use hierarchical partitioning of the agents, where a small set of UAVs $(\leq 10)$ are assigned to a single base-station and the operation consists of many base stations. With increase in number of nodes, the amount of data to be transmitted increases and a single receiver may not be able to handle such high traffic. Hence, the usual approach especially when imagery data need to be transmitted from UAVs is to assign a receiver to which a small set of UAVs communicate.

\section{CONClusions}

Cooperation between mobile computing agents enables them to optimize the computation and communication energy consumption, thereby increasing the system lifetime. We have devised an MINLP formulation that shows lower energy consumption by incorporating data aggregation and clustering schemes. The MINLP formulation is generic and we utilized this generality by validation on two data gathering applications, namely target tracking and mapping. We have studied the effect of different parameters on the MINLP decisionmaking. Simulation results show that the channel bandwidth 


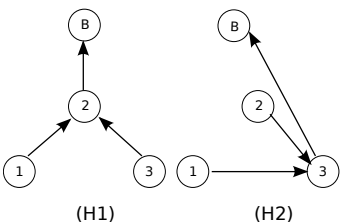

(a)

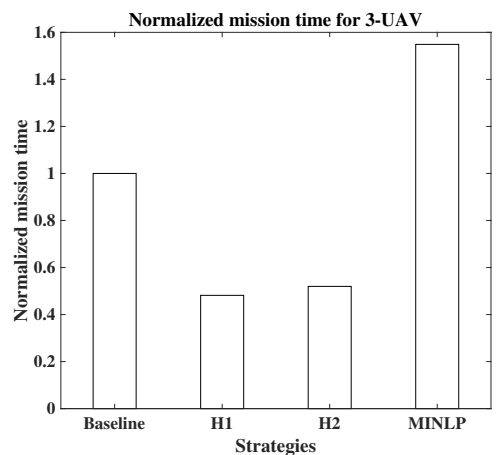

(b)

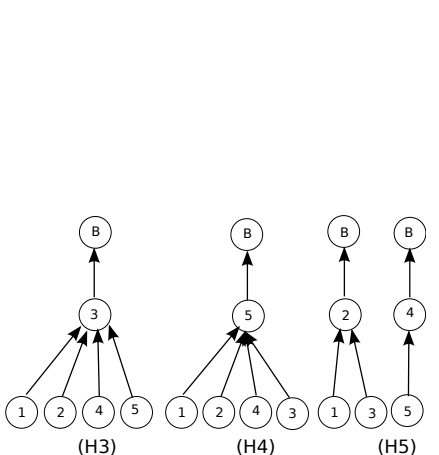

(c)

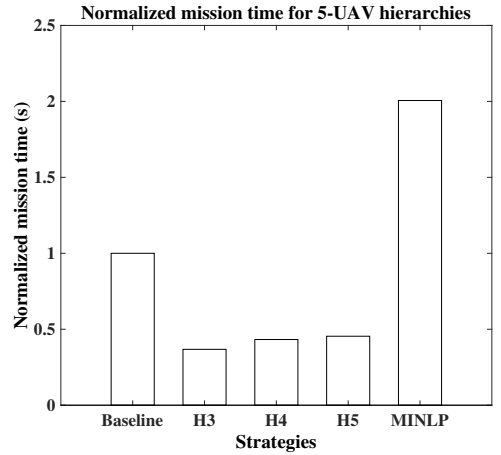

(d)

Fig. 10: (a) Two rigid structures $\mathrm{H} 1$ and $\mathrm{H} 2$. In $\mathrm{H} 1$, vehicle 2 transmits the data to the base station $\mathrm{B}$ and in $\mathrm{H} 2$ vehicle 3 transmits the information to the base station. (b) Performance of different strategies against the baseline strategy for a complete mission. (c) Three rigid structures H3, H4 and H5 for 5 agent scenario. (d) Normalized mission time of different strategies against the baseline strategy for a complete mission.

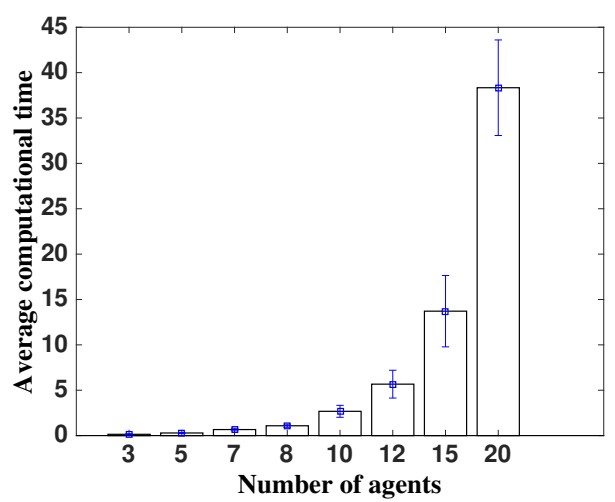

Fig. 11: Computational time taken to compute MINLP solution for increasing number of agents

has a direct impact on the energy saving scheme, i.e. sufficient bandwidth is necessary for an implementation of an intelligent information routing scheme.

The proposed MINLP formulation can be further extended to optimize the energy consumption of various units. One potential direction is to make a decision on when to communicate to the base station. Currently, we assume that the decision interval is fixed. However, depending on the amount of data, channel bandwidth and the transceiver energy properties, the decision cycle can be dynamically selected to optimize the overall energy consumption. Further, developing efficient algorithms to solve the MINLP problem as well as whether to implement the proposed framework in a centralized or distributed manner could be subjects for future work.

\section{REFERENCES}

[1] M. Bryson, A. Reid, F. Ramos, and S. Sukkarieh, "Airborne vision-based mapping and classification of large farmland environments," Journal of Field Robotics, vol. 27, no. 5, pp. 632-655, 2010.

[2] F. Nex and F. Remondino, "UAV for 3d mapping applications: a review," Applied Geomatics, vol. 6, no. 1, pp. 1-15, 2014.
[3] K. Anderson and K. J. Gaston, "Lightweight unmanned aerial vehicles will revolutionize spatial ecology," Frontiers in Ecology and the Environment, vol. 11, no. 3, pp. 138-146, 2013.

[4] L. Wallace, A. Lucieer, C. Watson, and D. Turner, "Development of a uav-lidar system with application to forest inventory," Remote Sensing, vol. 4, no. 6, pp. 1519-1543, 2012.

[5] G. Zhou, "Near real-time orthorectification and mosaic of small uav video flow for time-critical event response," IEEE Transactions on Geoscience and Remote Sensing, vol. 47, no. 3, pp. 739-747, March 2009.

[6] M. J. Allen, "Autonomous soaring for improved endurance of a small uninhabited air vehicle," in Proceedings of the AIAA Aerospace Sciences Meeting and Exhibit, Reno, Nevada, Jan 2005, pp. AIAA 2005-1025.

[7] https://www.fpvmodel.com

[8] http://www.rctigermotor.com/html/2013/Professional_0912/47.html

[9] https://www.raspberrypi.org

[10] www.hardkernel.com

[11] http://www.nvidia.com/object/jetson-tk1-embedded-dev-kit.html

[12] L. Doherty, B. A. Warneke, B. E. Boser, and K. Pister, "Energy and performance considerations for smart dust," International Journal on Parallel and Distributed Systems and Networks, vol. 4, no. 3, pp. 121133, 2001.

[13] Y. Chen and Q. Zhao, "On the lifetime of wireless sensor networks," IEEE Communications Letters, vol. 9, no. 11, pp. 976-978, Nov 2005.

[14] M. Bhardwaj and A. Chandrakasan, "Bounding the lifetime of sensor networks via optimal role assignments," Annual Joint Conference of the IEEE Computer and Communications (INFOCOMM), vol. 3, pp. 15871596, 2002.

[15] Y.-F. Wen and F.-S. Lin, "Energy-efficient data aggregation routing and duty-cycle scheduling in cluster-based sensor networks," IEEE Consumer Communications and Networking Conference, pp. 95-99, Jan 2007.

[16] H. Gupta, V. Navda, S. Das, and V. Chowdhary, "Efficient gathering of correlated data in sensor networks," ACM Transactions on Sensor Networks, vol. 4, no. 1, pp. 1-31, Feb. 2008.

[17] L. Xiang, J. Luo, and C. Rosenberg, "Compressed data aggregation: Energy-efficient and high-fidelity data collection," IEEE/ACM Transactions on Networking, vol. 21, no. 6, pp. 1722-1735, Dec 2013.

[18] N. Pantazis, S. Nikolidakis, and D. Vergados, "Energy-efficient routing protocols in wireless sensor networks: A survey," IEEE Communications Surveys Tutorials, vol. 15, no. 2, pp. 551-591, Feb 2013.

[19] W. Heinzelman, A. Chandrakasan, and H. Balakrishnan, "Energyefficient communication protocol for wireless microsensor networks," in Proceedings of the Annual Hawaii International Conference on System Sciences, Jan 2000.

[20] — , "An application-specific protocol architecture for wireless microsensor networks," in IEEE Transactions on Wireless Communications, vol. 1, no. 4, Oct 2002, pp. 660-670.

[21] S. Lindsey and C. Raghavendra, "Pegasis: Power-efficient gathering in sensor information systems," in IEEE Aerospace Conference, vol. 3 , 2002, pp. 3-1125-3-1130 vol.3. 
[22] J. Al-Karaki, R. Ul-Mustafa, and A. Kamal, "Data aggregation in wireless sensor networks - exact and approximate algorithms," in Workshop on High Performance Switching and Routing, 2004, pp. 241-245.

[23] O. Tekdas, V. Isler, J. H. Lim, and A. Terzis, "Using mobile robots to harvest data from sensor fields," IEEE Wireless Communications, vol. 16, no. 1, pp. 22-28, February 2009.

[24] R. Sugihara and R. Gupta, "Improving the data delivery latency in sensor networks with controlled mobility," in Distributed Computing in Sensor Systems, ser. Lecture Notes in Computer Science, S. Nikoletseas, B. Chlebus, D. Johnson, and B. Krishnamachari, Eds. Springer Berlin Heidelberg, 2008, vol. 5067, pp. 386-399.

[25] P. Sujit, D. Lucani, and J. Sousa, "Bridging cooperative sensing and route planning of autonomous vehicles," IEEE Journal on Selected Areas in Communications, vol. 30, no. 5, pp. 912-922, June 2012

[26] D.-T. Ho, E. Grtli, P. Sujit, T. Johansen, and J. Borges Sousa, "Clusterbased communication topology selection and uav path planning in wireless sensor networks," in International Conference on Unmanned Aircraft Systems, May 2013, pp. 59-68.

[27] H.-Y. Shum and L.-W. He, "Rendering with concentric mosaics," in Proceedings of the Annual Conference on Computer Graphics and Interactive Techniques, ser. SIGGRAPH '99. New York, NY, USA: ACM Press/Addison-Wesley Publishing Co., 1999, pp. 299-306.

[28] P. Zhan, K. Yu, and A. Swindlehurst, "Wireless relay communications using an unmanned aerial vehicle," in IEEE Workshop on Signal Processing Advances in Wireless Communications, July 2006, pp. 15.

[29] S. Ponda, L. Johnson, A. Kopeikin, H.-L. Choi, and J. How, "Distributed planning strategies to ensure network connectivity for dynamic heterogeneous teams," IEEE Journal on Selected Areas in Communications, vol. 30, no. 5, pp. 861-869, June 2012.

[30] A. Kassir, R. Fitch, and S. Sukkarieh, "Communication-aware information gathering with dynamic information flow," The International Journal of Robotics Research, vol. 34, no. 2, pp. 173-200, 2015.

[31] D. Pack, G. York, and R. Fierro, "Information-based cooperative control for multiple unmanned aerial vehicles," in IEEE International Conference on Networking, Sensing and Control, 2006, pp. 446-450.

[32] S. Quintero, F. Papi, D. Klein, L. Chisci, and J. Hespanha, "Optimal uav coordination for target tracking using dynamic programming," in IEEE Conference on Decision and Control, Dec 2010, pp. 4541-4546.

[33] L. Wang, F. Su, H. Zhu, and L. Shen, "Active sensing based cooperative target tracking using uavs in an urban area," in International Conference on Advanced Computer Control, vol. 2, March 2010, pp. 486-491.

[34] N. Adurthi and P. Singla, "Information driven optimal sensor control for efficient target localization and tracking," in American Control Conference, June 2014, pp. 610-615.

[35] M. Kalandros, L. Trailovic, L. Y. Pao, and Y. Bar-Shalom, "Tutorial on multisensor management and fusion algorithms for target tracking," American Control Conference, vol. 5, pp. 4734-4748, June 2004.

[36] M. Kalandros, "Covariance control for multisensor systems," IEEE Trans. Aerospace Electron. Systems, vol. 38, no. 4, pp. 1138-1157, Oct 2002.

[37] Q. Ling, Y. Fu, and Z. Tian, "Localized sensor management for multitarget tracking in wireless sensor networks," Information Fusion, vol. 12 pp. 194-201, 2011.

[38] O. Demigha, W.-K. Hidouci, and T. Ahmed, "On energy efficiency in collaborative target tracking in wireless sensor network: A review," IEEE Communications Surveys Tutorials, vol. 15, no. 3, pp. 1210-1222, MArch 2013.

[39] F. Zhao, J. Shin, and J. Reich, "Information-driven dynamic sensor collaboration," IEEE Signal Processing Magazine, vol. 19, no. 2, pp. 61-72, Mar 2002.

[40] R. Olfati-Saber, "Distributed tracking for mobile sensor networks with information-driven mobility," in Proc. of the American Control Conference, July 2007, pp. 4606-4612.

[41] J. Lin, W. Xiao, F. Lewis, and L. Xie, "Energy-efficient distributed adaptive multisensor scheduling for target tracking in wireless sensor networks," IEEE Transactions on Instrumentation and Measurement, vol. 58, no. 6, pp. 1886-1896, June 2009.

[42] R. Rajagopalan and P. Varshney, "Data-aggregation techniques in sensor networks: A survey," IEEE Communications Surveys Tutorials, vol. 8 , no. 4, pp. 48-63, 2006.

[43] Y. Hamouda and C. Phillips, "Metadata-based adaptive sampling for energy-efficient collaborative target tracking in wireless sensor networks," in IEEE International Conference on Computer and Information Technology, June 2010, pp. 313-320.
[44] F. Delicato, F. Protti, L. Pirmez, and J. F. de Rezende, "An efficient heuristic for selecting active nodes in wireless sensor networks," Computer Networks, vol. 50, no. 18, pp. 3701 - 3720, 2006.

[45] Z. Wang, W. Lou, Z. Wang, J. Ma, and H. Chen, "A novel mobility management scheme for target tracking in cluster-based sensor networks," in Distributed Computing in Sensor Systems, ser. Lecture Notes in Computer Science, R. Rajaraman, T. Moscibroda, A. Dunkels, and A. Scaglione, Eds. Springer Berlin Heidelberg, 2010, vol. 6131, pp. 172-186.

[46] H. Eisenbeiss, "A mini unmanned aerial vehicle (uav): system overview and image acquisition," International Archives of Photogrammetry. Remote Sensing and Spatial Information Sciences, vol. 36, no. 5/W1, 2004.

[47] F. Nex and F. Remondino, "Uav for 3d mapping applications: a review," Applied Geomatics, vol. 6, no. 1, pp. 1-15, 2014.

[48] K. M. Fornace, C. J. Drakeley, T. William, F. Espino, and J. Cox, "Mapping infectious disease landscapes: unmanned aerial vehicles and epidemiology," Trends in parasitology, vol. 30, no. 11, pp. 514-519, 2014.

[49] C. Di Franco and G. Buttazzo, "Energy-aware coverage path planning of uavs," in IEEE International Conference on Autonomous Robot Systems and Competitions, 2015, pp. 111-117.

[50] J. J. Chung, N. Lawrance, S. K. Gan, Z. Xu, R. Fitch, and S. Sukkarieh, "Variable density prm waypoint generation and connection radii for energy-efficient flight through wind fields," in IEEE International Conference on Robotics and Automation, no. 3, 2015, pp. 2-4.

[51] K. Cesare, R. Skeele, S.-H. Yoo, Y. Zhang, and G. Hollinger, "Multiuav exploration with limited communication and battery," in IEEE International Conference on Robotics and Automation, 2015, pp. 22302235.

[52] A. Noth, "Design of solar powered airplanes for continuous flight," Ph.D. dissertation, ETH Zürich, 2008.

[53] M. Patel, S. Venkatesan, and D. Weiner, "Role assignment for data aggregation in wireless sensor networks," International Conference on Advanced Information Networking and Applications Workshops, vol. 2, pp. 390-395, May 2007.

[54] J. Zhu and S. Papavassiliou, "On the energy-efficient organization and the lifetime of multi-hop sensor networks," IEEE Communications Letters, vol. 7, no. 11, pp. 537-539, Nov 2003.

[55] Y. Tian, J. Boangoat, E. Ekici, and F. Ozguner, "Real-time task mapping and scheduling for collaborative in-network processing in dvs-enabled wireless sensor networks," IEEE International Parallel and Distributed Processing Symposium, April 2006.

[56] B. Zeng, J. Wei, and H. Liu, "Research of optimal task scheduling for distributed real-time embedded systems," International Conference on Embedded Software and Systems, pp. 77-84, July 2008.

[57] MATLAB, version 7.14.0.739 (R2012a). Natick, Massachusetts: The MathWorks Inc., 2012.

[58] J. Currie and D. I. Wilson, "OPTI: Lowering the Barrier Between Open Source Optimizers and the Industrial MATLAB User," in Foundations of Computer-Aided Process Operations, N. Sahinidis and J. Pinto, Eds., Savannah, Georgia, USA, 8-11 January 2012.

[59] T. Achterberg, "SCIP: Solving constraint integer programs," Mathematical Programming Computation, vol. 1, no. 1, pp. 1-41, July 2009.

[60] T. Vercauteren and X. Wang, "Decentralized sigma-point information filters for target tracking in collaborative sensor networks," IEEE Transactions on Signal Processing, vol. 53, no. 8, pp. 2997-3009, Aug 2005.

[61] S. Kim, H. Oh, J. Suk, and A. Tsourdos, "Coordinated trajectory planning for efficient communication relay using multiple UAVs," Control Engineering Practice, vol. 29, pp. 42 - 49, 2014.

[62] B. P. Hudzietz and S. Saripalli, "An experimental evaluation of 3d terrain mapping with an autonomous helicopter," in Conference on Unmanned Aerial Vehicle in Geomatics, 2011, pp. 137-142.

[63] P. Sujit, S. Saripalli, and J. Borges Sousa, "Unmanned aerial vehicle path following: A survey and analysis of algorithms for fixed-wing unmanned aerial vehicless," IEEE Control Systems, vol. 34, no. 1, pp. 42-59, 2014.

[64] D. Nelson, D. Barber, T. McLain, and R. Beard, "Vector field path following for miniature air vehicles," IEEE Transactions on Robotics, vol. 23 , no. 3, pp. 519-529, 2007 


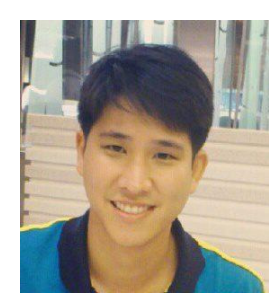

Mason Thammawichai received the BS degree in Computer Engineering from University of Wisconsin-Madison, USA and the MSc in Avionic Systems from University of Sheffield, UK. He completed $\mathrm{PhD}$ from Imperial College London, UK in 2016. Currently, he is with the His main areas of research are real-time scheduling, mathematical optimization and optimal control.

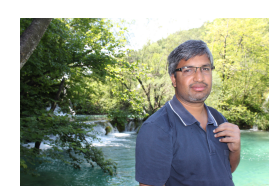

Sujit P.Baliyarasimhuni is an Assistant Professor at the Indraprastha Institute of Information Technology, New Delhi, India and lecturer at Loughborough University, UK. He received the bachelor's degree from Bangalore University, India, Master's degree from Visveswaraya Technological University, India, and $\mathrm{Ph} . \mathrm{D}$. from the Indian Institute of Science, Bangalore, India. His research interests include unmanned aerial and underwater vehicles, multi-robot systems, and human-robot interaction.

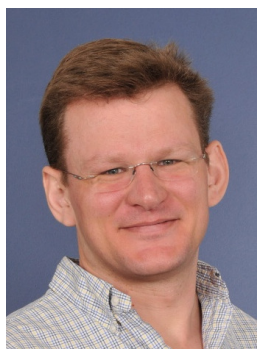

Eric C. Kerrigan (S'94-M'02) received a PhD from the University of Cambridge in 2001 and has been a faculty member at Imperial College London since 2006. His research is on efficient numerical methods and computing architectures for solving advanced optimization, control and estimation problems in aerospace, renewable energy and computing systems. He is on the IEEE Control Systems Society Conference Editorial Board and is an associate editor of the IEEE Transactions on Control Systems Technology and Control Engineering Practice.

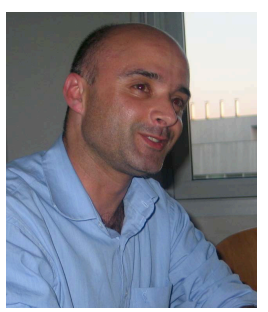

João B. Sousa is an Assistant Professor with the Electrical and Computer Engineering Department at Porto University. He is the head of the Underwater Systems and Technologies Laboratory. His research interests include unmanned vehicles, control architectures, control, and coordination. He has been leading the design, implementation, and deployment of advanced unmanned air and ocean vehicle systems in projects funded by the Portuguese Science Foundation (FCT), the Portuguese Ministry of Defense, the Portuguese Innovation Agency (ADI), the European Union (FP6/FP7/H2022), NATO and, in the United States of America, the Office of Naval Research and DARPA. In 2006 the laboratory received the national BES Innovation National Award for the design of the Light Autonomous Underwater Vehicle. In 2007 he received an outstanding teaching award from Porto University. He has authored approximately 240 scientific papers. 